\title{
GLOBAL CONTINUOUS SOLUTIONS OF HYPERBOLIC SYSTEMS OF QUASI-LINEAR EQUATIONS ${ }^{1}$
}

\author{
BY J. L. JOHNSON
}

Communicated by Jürgen Moser, May 16, 1967

Recently there have appeared a number of results on global solutions of the Cauchy problem for hyperbolic systems of quasi-linear equations [2], [3], [4], [5]. These solutions are in general discontinuous. In certain cases, however, such as the interaction of two rarefaction waves in gas dynamics, it is known that the Cauchy problem has a global continuous solution [1, pp. 191-197]. In this announcement we outline a proof that a global continuous solution exists and is unique for a two-dimensional system provided the Riemann invariants associated with the initial data satisfy certain monotonicity and continuity conditions.

Let $\lambda^{+}(r, s), \lambda^{-}(r, s)$ be $C^{1}$ real-valued functions on a domain $D \subset R_{2}$, with

$$
\lambda^{+}(r, s)>\lambda-(r, s), \quad \partial \lambda^{+}(r, s) / \partial r>0, \quad \partial \lambda^{-}(r, s) / \partial s>0
$$

for $(r, s) \in D$. Consider the two-dimensional system of quasi-linear equations in Riemann invariant form

$$
r_{t}+\lambda^{+}(r, s) r_{x}=0, \quad s_{t}+\lambda^{-}(r, s) s_{x}=0
$$

where $r(t, x)$ and $s(t, x)$ are real-valued functions of two scalar variables. We seek a solution of the Cauchy problem in the halfplane $\left\{(t, x) \in R_{2}: t \geqq 0\right\}$ with initial conditions

$$
r(0, x)=r^{0}(x), \quad s(0, x)=s^{0}(x), \quad-\infty<x<+\infty .
$$

Let $G_{T}=\left\{(t, x) \in R_{2}: 0 \leqq t<T\right\}$ for $0<T \leqq+\infty$. A pair of Lipschitz continuous functions $(r(t, x), s(t, x)),(t, x) \in G_{T}$, is called a Lipschitz continuous solution of the Cauchy problem (2), (3) if $r(t, x)$ is constant on the integral curves

$$
x^{\prime}(t)=\lambda^{+}(r(t, x), s(t, x)),
$$

$s(t, x)$ is constant on the integral curves

$$
x^{\prime}(t)=\lambda-(r(t, x), s(t, x)),
$$

${ }^{1}$ These results are part of the author's Ph.D. thesis written under Joel A. Smoller at the University of Michigan. 
and $r(0, x), s(0, x)$ satisfy the initial conditions (3). The pair $(r(t, x)$, $s(t, x))$ is called a global Lipschitz continuous solution of (2), (3) if the functions are defined and Lipschitz continuous on $G_{\infty}$.

Theorem 1. If $r^{0}(x), s^{0}(x),-\infty<x<+\infty$, are bounded, Lipschitz continuous, and nondecreasing, satisfying

$$
\left[r^{0}(-\infty), r^{0}(+\infty)\right] \times\left[s^{0}(-\infty), s^{0}(+\infty)\right] \subset D,
$$

then the Cauchy problem (2), (3) with initial functions $r^{0}(x), s^{0}(x)$, $-\infty<x<+\infty$, has a global Lipschitz continuous solution which takes its values in the rectangle (6).

Outline of Proof for Theorem 1. For each finite subset $A$ of $R_{1}$ we construct an approximate solution $(r(t, x ; A), s(t, x ; A)),(t, x)$ $\in G_{\infty}$, with the property that $r(t, x ; A)$ is constant on curves of the form (4) and $s(t, x ; A)$ is constant on a finite number of curves of the form (5). Using condition (1) and the assumed properties of the initial functions, we show that $r(t, x ; A)$ is Lipschitz continuous in $G_{\infty}$ with Lipschitz constant independent of $t, x$, and $A$. If $\left\{B_{n}: n=1,2, \cdots\right\}$ is an increasing sequence of finite sets whose union is dense in $R_{1}$, then by the Ascoli theorem the sequence of functions $r\left(t, x ; B_{n}\right)$ contains a subsequence converging to a Lipschitz continuous function $r(t, x)$. Having this function, we construct $s(t, x),(t, x) \in G_{\infty}$, so that the pair $(r(t, x), s(t, x)),(t, x) \in G_{\infty}$, is a global Lipschitz continuous solution of (2), (3).

We have also obtained the following result regarding the dependence of Lipschitz continuous solutions on initial data.

Theorem 2. Let $r_{i}^{0}(x), s_{i}^{0}(x),-\infty<x<+\infty, i=1,2$, be bounded real-valued functions with

$$
a_{i} \leqq r_{i}^{0}(x) \leqq b_{i}, \quad c_{i} \leqq s_{i}^{0}(x) \leqq d_{i}, \quad-\infty<x<+\infty,
$$

and suppose that $\left[a_{i}, b_{i}\right] \times\left[c_{i}, d_{i}\right] \subset D, i=1,2$. Let

$$
m=\sup _{-\infty<x<+\infty}\left(\left|r_{1}^{0}(x)-r_{2}^{0}(x)\right|+\left|s_{1}^{0}(x)-s_{2}^{0}(x)\right|\right) .
$$

If $\left(r_{i}(t, x), s_{i}(t, x)\right),(t, x) \in G_{T}$, is a Lipschitz continuous solution of the Cauchy problem for the equations (2) with initial vector $\left(r_{i}^{0}(x), s\left({ }_{i}^{0} x\right)\right)$, $-\infty<x<+\infty, i=1,2$, then there is a constant $L(T)$ such that

$$
\sup _{(t, x) \in G_{T}}\left(\left|r_{1}(t, x)-r_{2}(t, x)\right|+\left|s_{1}(t, x)-s_{2}(t, x)\right|\right) \leqq m L(T) .
$$


It follows easily from this that Lipschitz continuous solutions are unique.

Theorems 1 and 2, together with results of Lax [3], can be applied to the conservation law

$$
u_{\iota}+(p(v))_{x}=0, \quad v_{t}-u_{x}=0
$$

to yield the following corollaries.

Corollary 1. Let $p(v) \in C^{2}$ on the halfine $(v>0)$ with $p^{\prime}(v)<0$, $p^{\prime \prime}(v)>0$ and $\int_{1}^{\infty}\left[-p^{\prime}(v)\right]^{1 / 2} d v=\infty$. If the functions $u^{0}(x), v^{0}(x)$, $-\infty<x<+\infty$, are bounded and Lipschitz continuous, with $v^{0}(x)$ positive and bounded away from 0 , and satisfy

$$
u^{0}\left(x_{2}\right)-u^{0}\left(x_{1}\right) \geqq\left|\int_{v^{0}\left(x_{1}\right)}^{v^{0}\left(x_{2}\right)}\left[-p^{\prime}(v)\right]^{1 / 2} d v\right| \quad \text { for } x_{2}>x_{1},
$$

then the Cauchy problem for the equations (7) with initial vector $\left(u^{0}(x)\right.$, $\left.v^{0}(x)\right)$ has a unique Lipschitz continuous weak solution.

Corollary 2. Let $p(v)$ be as in Corollary 1 , and let $u^{0}(x), v^{0}(x)$, $-\infty<x<+\infty$, be bounded and piecewise constant real-valued functions, with $v^{0}(x)$ positive and bounded away from 0 , which satisfy (8). If the set $A$ of discontinuities of the vector function $\left(u^{0}(x), v^{0}(x)\right)$ has the property

$$
\inf \{|a-b|: a, b \in A, a \neq b\}>0,
$$

then the Cauchy problem for the system (7) with initial vector $\left(u^{0}(x)\right.$, $\left.v^{0}(x)\right)$ has a solution which is Lipschitz continuous in each of the sets $\left\{(t, x) \in G_{\infty}: t>t_{0}\right\}$, for $t_{0}>0$.

Corollary 2 provides a solution for the interaction of simple waves centered on the line $(t=0)$.

\section{REFERENCES}

1. R. Courant and K. O. Friedrichs, Supersonic flow and shock waves, Interscience, New York, 1948.

2. James Glimm, Solutions in the large for nonlinear hyperbolic systems of equations, Comm. Pure Appl. Math. 18 (1965), 697-715.

3. P. D. Lax, Hyperbolic systems of conservation lares. II, Comm. Pure Appl. Math. 10 (1957), 537-566.

4. B. L. RoždestvenskiY, Construction of discontinuous solutions to systems of quasilinear equations. I, II, Ž. Vyčisl. Mat. i Mat. Fiz. 2 (1962), 1019-1043; 3 (1963), 79-98.

5. Zhang Tong and Guo Yu-fa, $A$ class of initial value problems for systems of aerodynamic equations, Acta Math. Sinica 15 (1965), no. 3, 386-396; English transl., Chinese Math. 7 (1965), 90-101.

UNIVERSITY OF MICHIGAN 